\title{
Implizite Bedeutung in sozialen Netzwerken Stärkung des gesellschaftlich-demokratischen Bewusst- seins im Französisch als Fremdspracheunterricht
}

\author{
Dr. Nicola Brocca, Universität Innsbruck
}

\section{Einleitung}

Am Anfang der 2010er Jahre habe ich in einer Gemeinschaftsschule in BerlinKreuzberg Französisch unterrichtet. Smartphones verschafften sich in dieser Zeit den Weg in die Tasche der SchülerInnen und aus den Schulranzen piepste es während des Unterrichts immer häufiger. Da die Smartphones nicht mehr mit Verboten einzudämmen waren, beschlossen wir Lehrkräfte ihre Nutzung zumindest zu regulieren. Bei einer Schulkonferenz entschied das Kollegium einen Raum für freie Smartphonenutzung zu schaffen und die Anwendung von internetfähigen Endgeräten bei Bedarf im Unterricht $\mathrm{zu}$ erlauben. Die Smartphone Ecke wurde von SchülerInnen sehr positiv begrüßt: In den Pausen versammelten sich viele SchülerInnen dort auf einigen Sitzsteinen im Schulhof. Einige SchülerInnen spielten ,Grand Theft Auto' und holten sich auf YouTube Tipps, um sich zu verbessern. Andere nahmen Videoclips mit musical.ly auf und teilten sie auf Facebook. Jedoch blieb manchmal das, was die SchülerInnen mit ihren Geräten machten, uns Lehrkräften verborgen. Dies änderte sich, als einige Wochen später wiederholt Meldungen bezüglich Konflikten und sogar Straftaten, die auf den ungeregelten und unkritischen Umgang mit Social Network Sites (SNSs) zurückzuführen waren, unsere Schule erreichten. Seitdem

Kapelari, Suzanne (Hg.), Vierte ,Tagung der Fachdidaktik“ 2019:

„Interdisziplinäre fachdidaktische Diskurse zur Bildung für nachhaltige Entwicklung“

(C) 2020 innsbruck university press, ISBN 978-3-99106-019-2, DOI 10.15203/99106-019-2 
wurde die handyfreie Zone abgeschafft und ein gänzliches Verbot von Mobiltelefonen wiedereingeführt. Damit spielte sich die Mediennutzung nicht mehr unter den Augen der Lehrkräfte ab, und die schulische Bildung legte ihren Einfluss auf was die SchülerInnen in SNSs lasen, lernten und weiterleiteten ab.

Die Reaktion dieser Berliner Schule war kein Einzelfall; allgemein zeigen sich Schulen sehr zurückhaltend gegenüber internetfähigen Mobilgeräten: Laut der JIM-Studie (2016) darf nur ein Drittel der befragten deutschen SchülerInnen das Handy in der Schule ausschließlich in den Pausen nutzen. Die Tendenz, internetfähige mobile Endgeräte im Unterricht einzusetzen, ist jedoch rasch gestiegen. 2015 durften nur 7\% der SchülerInnen ihr Handy gezielt im Schulunterricht einsetzen. 2016 belief sich dieser Anteil bereits auf 22\% (JIMStudie, 2015, 2016). Besser erscheint die Haltung gegenüber Smartphones oder Tablets in Österreich: Nach Angabe des Bundesministeriums für Bildung, Wissenschaft und Forschung (BBWF) wird an rund zwei Dritteln der NMS, AHS und BMHS ${ }^{1}$ mit eigenen Geräten (Smartphones, Tablets oder Notebooks) bei Bedarf im Unterricht gearbeitet (Bundesministerium für Bildung Wissenschaft und Forschung, 2019, S. 1). Die Praxis außerhalb der Schule sieht demgegenüber jedoch anders aus: Über drei Stunden verbringen deutsche Jugendliche durchschnittlich jeden Tag nur mit SNS (DAK-Gesundheit \& UKE, 2018; JIM, 2017). Neben der Befriedigung von sozialen Bedürfnissen ist das Interesse für aktuelle politische Themen etwas, das die Jugendlichen in SNS bewegt (JIM, 2017): 34\% der 18-19-Jährigen geben an, primär in Facebook Nachrichten zum aktuellen Weltgeschehen zu suchen (ebd.).

Das zeigt, dass SNS sich als Online-Nachrichtenmedien etabliert haben. Die Auswirkungen dieser Entwicklung sind noch nicht vollständig verstanden worden, insbesondere wenn es um die Rolle der SNS bei der Gestaltung der Überzeugungen der Menschen in einem zunehmend polarisierten Europa geht. SNS sind für PolitikerInnen von besonderem Wert, da sie direkter und in allgegenwärtigen Situationen mit ihren AnhängerInnen kommunizieren können als zuvor.

1 NMS steht für Neue Mittelschule, AHS für allgemein bildende höhere Schule und BMHS für Berufsbildende mittlere und höhere Schule.

Kapelari, Suzanne (Hg.), Vierte „Tagung der Fachdidaktik“ 2019:

„Interdisziplinäre fachdidaktische Diskurse zur Bildung für nachhaltige Entwicklung“

(C) 2020 innsbruck university press, ISBN 978-3-99106-019-2, DOI 10.15203/99106-019-2 
Das Verstehen von politischen Botschaften in SNS und die kritische Auseinandersetzung mit ihnen kann als übergreifende Kompetenz für die Realisierung der Ziele für die Nachhaltige Entwicklung (SDGs) gesehen werden. Dabei findet sich diese Kompetenz in erster Linie im Ziel der hochwertigen Bildung (Ziel 4 SDGs). Darüberhinausgehend kann sie auch der Förderung von friedlichen und gerechten Institutionen (Ziel 16 SDGs) und inklusiven Gesellschaften (Ziel 10 SDGs) zugeordnet werden (siehe Rieckmann in diesem Band).

Die SDGs und die auf ihnen basierenden Ziele der Agenda 2030 (Vereinte Nationen, 2015) überlassen den nationalen Staaten die Umsetzung der Ziele. Da in Europa demokratische Institutionen sowie der gesellschaftliche Zusammenhalt in den letzten Jahren immer wieder gefährdet $\operatorname{sind}^{2}$, sollte ihre Verstärkung und ihr Schutz auch im Rahmen der SDGs von prioritärer Wichtigkeit werden.

Eine Bildungsstrategie, die die SchülerInnen im Sinne der Ziele der SDGs bilden möchte, muss die Förderung der Demokratie und einer integrativen und kohäsiven Gesellschaft berücksichtigen. Dazu gehört auch die Kompetenz jüngerer Menschen, in der Lage zu sein, sich aus Fakten ein Meinungsbild zu verschaffen und Manipulationsversuche zu erkennen. Wenn sich junge Menschen immer öfter auf Basis von sozialen Medien ihre Meinung bilden, kann das Bildungssystem sich zukünftigen Herausforderungen der Kommunikation online nicht verschließen. Das Strategiepapier der ständigen Konferenz der Kultusminister der Länder in der Bundesrepublik Deutschland zur „Bildung in der digitalen Welt“ (KMK, 2017) sieht vor, den Bildungserfolg noch stärker darauf auszurichten, „Fakten, Prozesse, Entwicklungen einerseits einzuordnen und zu verknüpfen, andererseits zu bewerten und dazu Stellung zu nehmen“ (S.13). Hierzu kann eine bewusste und kritische Anwendung von Internetressourcen einen wesentlichen Beitrag leisten. Auch im österreichischen bildungspolitischen Diskurs wird die Verschränkung zwischen Informations-

2 Z.B. der Aufstieg von EU- und minderheitsfeindlichen Parteien oder der Brexit.

Kapelari, Suzanne (Hg.), Vierte „Tagung der Fachdidaktik“ 2019:

„Interdisziplinäre fachdidaktische Diskurse zur Bildung für nachhaltige Entwicklung“

(C) 2020 innsbruck university press, ISBN 978-3-99106-019-2, DOI 10.15203/99106-019-2 
kompetenzen und sozial-politischem Zusammenhalt betont: Der ,produktive Umgang mit Medien für eine kritische, bewusste [...] staatsbürgerliche Teilhabe“ (S. 97) wird als Ziel der Medienerziehung im Nationalen Bildungsbericht Österreich definiert (Baumgartner, Brandhofer, Ebner, Gradinger \& Korte, 2015).

Dieser Aufsatz beschäftigt sich mit der Frage, welchen Beitrag die Sprachwissenschaft und die Fremdsprachendidaktik leisten können, um Ziele in der Medienbildung zu verfolgen, die eine demokratische und integrative Gesellschaft fördern. Dabei wird auf theoretische Erkenntnisse der Grice'schen Pragmalinguistik (Grice, 1975) zurückgegriffen und daraus eine Lerneinheit mit Content and Language Integrated Learning (CLIL) Ansatz skizziert. Dieser Beitrag argumentiert, warum - trotz gewisser Hürden - die Fähigkeit, soziale Netzwerke kritisch zu lesen, eine grundlegende Kompetenz für eine Bildung, die die SDG fördert, darstellt und warum es daher sinnvoll ist, diese Kompetenzen (auch) im Fremdsprachenunterricht zu vermitteln. Der Beitrag ist folgendermaßen aufgebaut: In Abschnitt zwei wird die Präsenz der kritischen Medienbildung in Lehrplänen untersucht. Abschnitt drei behandelt die Rolle der Pragmalinguistik als Mittel des kritischen Textverständnisses, während der vierte Abschnitt die medialen und sprachlichen Kompetenzen zusammenführt. Abschießend wird in Abschnitt fünf eine Zwischenbilanz zur Validität und Durchführbarkeit der vorgestellten Materialien gezogen. Im Anhang finden sich Unterrichtsmaterialien, die exemplarisch zeigen sollen, wie diese Überlegungen in die Praxis des Fremdsprachunterrichts übertragen werden können.

\section{Medienbildung und gesellschaftliche Teilhabe im Lehrplan für Fremdsprachen}

$\mathrm{Zu}$ den Zielen der Vereinten Nationen für eine Nachhaltige Entwicklung zählt die Förderung friedlicher und integrativer Gesellschaften und der Aufbau effektiver, rechenschaftspflichtiger und integrativer Institutionen (Ziel 16 SDGs). $\mathrm{Zu}$ diesem Ziel gehören die uneingeschränkte gesellschaftliche 
Teilhabe, die Entwicklung effektiver, rechenschaftspflichtiger und transparenter Institutionen (Ziel 16.6 SDGs) sowie die Sicherstellung einer reaktionsschnellen, integrativen, partizipativen und repräsentativen Entscheidungsfindung (Ziel 16.7 SDGs) (Vereinte Nationen, 2015; siehe auch Rieckmann in diesem Band).

Ein Echo dieser Einstellung ist auch in den Lehrplänen der österreichischen allgemeinbildenden höheren Schulen zu finden: Zu den Aufgabenbereichen des schulischen Fremdsprachenunterrichts gehört demnach, das

wertorientierte Denken und Handeln im politischen, sozialen, wirtschaftlichen, kulturellen und weltanschaulichen Umfeld zu fördern. [...] Der Erwerb einer Fremdsprache dient u.a. [...] der bewussten Auseinandersetzung mit [...] Wahrnehmungen und Wertungen [und soll] zum [...] friedlichen Zusammenleben beitragen. (Bundesministerium für Unterricht und Kunst, 1985: Fassung vom 01.09.2018) (Hervorhebungen durch den Verfasser)

Die Lehrpläne nehmen keine Einschränkung der Medienarten vor, die in den jeweiligen Fächern zum Erreichen der Lernziele eingesetzt werden können:

das selbstständige Erschließen und Erfassen schriftlicher fremdsprachlicher Texte verschiedener Art mit Hilfe angemessener Lesestrategien [...] die produktive schriftliche Anwendung der erworbenen Sprachmittel in adressatenadäquater und mediengerechter, d.h. der jeweiligen Textsorte entsprechender, Form. (Bundesministerium für Unterricht und Kunst, 1985: Fassung vom 01.09.2018) (Hervorhebungen durch den Verfasser)

Wie in anderen EU Ländern (Brocca, Borowiec, \& Masia, 2020: S.83) wird die Einführung neuer Technologien als didaktische Medien explizit aufgrund der anzustrebenden Aktualität und Anschaulichkeit empfohlen:

Kapelari, Suzanne (Hg.), Vierte „Tagung der Fachdidaktik“ 2019:

„Interdisziplinäre fachdidaktische Diskurse zur Bildung für nachhaltige Entwicklung“

(C) 2020 innsbruck university press, ISBN 978-3-99106-019-2, DOI 10.15203/99106-019-2 
Die Materialien und Medien, die im Unterricht eingesetzt werden, haben möglichst aktuell und anschaulich zu sein, um die Schülerinnen und Schüler zu aktiver Mitarbeit anzuregen [...]. Den neuen Technologien kommt verstärkt Bedeutung zu. (Herstellen von Bezügen zur Lebenswelt). (Bundesministerium für Unterricht und Kunst, 1985: Fassung vom 01.09.2018)

Zur Arbeit mit neuen Technologien wird nicht nur geraten, um rezeptive Kompetenzen $\mathrm{zu}$ fördern, sondern auch, um produktive Kompetenzen und Lernautonomie zu stimulieren: „Die Erstellung eigenständiger Arbeiten mit Mitteln der Informationstechnologie ist anzuregen“ (Bundesministerium für Unterricht und Kunst, 1985 Fassung vom 01.09.2018). Die Einführung von digitalen Medien beschränkt sich nicht auf deren Rolle als didaktische Mittel. Laut Desiderata des österreichischen Bundesministeriums für Unterricht und Kunst können digitale Medien (und somit auch SNS) selbst Thema des Unterrichts werden, wie im folgenden Zitat deutlich wird:

Zur Erlangung eines möglichst umfassenden lexikalischen Repertoires (Handlungsfähigkeit/ Kommunikationsfähigkeit) sind verschiedenste Themenbereiche zu bearbeiten [...]; Rolle der Medien; Einstellungen und Werte; Zusammenleben; aktuelle soziale, wirtschaftliche, technische und politische Entwicklungen. (Bundesministerium für Unterricht und Kunst, 1985: Fassung vom 01.09.2018) (Hervorhebungen durch den Verfasser)

Obwohl die Förderung der Nutzung von digitalen Medien und OnlineInformationsdiensten im Unterricht an zahlreichen Stellen betont wird, bleiben die Ziele, die Inhalte und die didaktischen Formen der digitalen Medien meist nur vage formuliert (Bundesministerium für Bildung, Wissenschaft und Forschung, 2019: 2). Das Potential der Nutzung von digitalen Technologien, um das politische und soziale Umfeld der SchülerInnen zu stärken und zudem (meta)sprachliche Kompetenzen der SchülerInnen zu fördern, wird ebenso nicht ausgeschöpft. 
Schließlich ist auch die Notwendigkeit einer kritischen Auseinandersetzung mit den digitalen Medien aus den Lehrplänen nicht ersichtlich. Es werden zwar indirekt pädagogische, inhaltliche und technische Bereiche angesprochen (Mishra \& Koehler, 2006), nicht aber übergeordnete Kompetenzen für eine digitalisierte Gesellschaft ins Auge gefasst (Baumgartner et al., 2016; KMK, 2017). Damit geht ein Teil der Sinnhaftigkeit eines Medienbezugs verloren und der Fokus auf digitale Technologien läuft Gefahr, als Modeerscheinung abgetan zu werden. Daten bezüglich der Nutzung von (sozialen) Medien seitens der neuen Generation belegen das Gegenteil. Ein Blick auf den stetig wachsenden Gebrauch von SNS seitens Jugendlicher (Bitkom, 2019) spricht für die Nachhaltigkeit des Themas und ruft zum nötigen Einsatz der Bildungsbeauftragten auf. Der Zusammenhang von Gebrauch von SNS seitens Jugendlicher und ihrer Meinungsbildung ist ebenso deutlich: Neben der Lösung persönlicher Probleme (62\%) geben Jugendliche an, über SNS am meisten auf Informationen zum aktuellen Weltgeschehen (56\%) zuzugreifen. Auch Umweltthemen (40\%) sowie Bundes- und Weltpolitik (39\%) sind unter den meistgesuchten Bereichen (JIM-Studie, 2017). Wenn Jugendliche explizit gefragt werden, beurteilen sie die Glaubwürdigkeit der Informationen online konservativer als jene in Print-Medien oder TV: Nur 16\% würden im Falle widersprüchlicher Berichte der Berichterstattung im Internet eher Glauben schenken, und das trotz Steigerung der Nutzung digitaler Medien (JIM-Studie, 2005-2014). Informationen in SNS werden aber selten als ,Berichte“ wahrgenommen, da sie aus vermeintlich vertrauten und glaubwürdigen Quellen stammen und deswegen häufig Freiraum für die Zirkulation von ungenauen oder schlicht nicht fundierten Informationen lassen, die ohne kritisches Filtern angenommen werden.

Der divulgative technische Diskurs spricht von Echo Chambers (DiFonzo, 2011) oder von Filter Bubbles (Pariser, 2011) und deutet darauf hin, dass Algorithmen, personalisierte Suchergebnisse bei der Internetrecherche, sowie die ideologische Segregation in SNS die Meinungsbildung der Jugendlichen beschränken bzw. radikalisieren und die demokratische Gesellschaft polarisieren (Sunstein, 2017). Selbst wenn diese Hypothesen sich als überschätzt nachweisen lassen (Barberá, Jost, Nagler, Tucker, \& Bonneau,

Kapelari, Suzanne (Hg.), Vierte „Tagung der Fachdidaktik“ 2019:

„Interdisziplinäre fachdidaktische Diskurse zur Bildung für nachhaltige Entwicklung“

(C) 2020 innsbruck university press, ISBN 978-3-99106-019-2, DOI 10.15203/99106-019-2 
2015; Dubois \& Blank, 2018), und die genauen Einflüsse der SNS auf Jugendliche noch nicht quantitativ belegt sind (Mills, 2016), ist die Gefahr einer einfachen Manipulation (Vergeer, 2013) durch unbewusste (und daher unkritische) Informationsselektion in SNS zu groß, um von Bildungsträgern ignoriert zu werden.

Der bewusste Einsatz von pragmalinguistisch-basierten Lesekompetenzen kann die Einflüsse einer persuasiven Kommunikation minimieren und es damit Jugendlichen ermöglichen, Informationsquellen auf der Basis der eigenen Kritikfähigkeit zu lesen (Brocca \& Masia, in Vorbereitung). Somit könnten Lehrkräfte SchülerInnen dabei fördern, sich reflektiert zu informieren und mündige BürgerInnen in einer digitalisierten Welt zu werden.

\section{Die Rolle der Pragmalinguistik für das Verständnis von Überzeugungsstrategien}

Der Informationsfluss in SNS unterliegt vielfältigen Faktoren wie starkem sozialen Druck, Knappheit, Multimodalität, Anonymität der Sender und Empfänger, etc. Um das Spektrum der Variablen zu reduzieren, wird dieser Beitrag sich ausschließlich mit Nachrichten über Twitter als prototypische SNS auseinandersetzen. Twitter stellt zwar bei Jugendlichen keine so wichtige Informationsquelle wie andere SNS (z.B. WhatsApp oder Instagram) dar (vgl. JIM-Studie, 2015: 32; Jugend-Internet-Monitor, 2019), trotzdem erweist sich dieses Medium für didaktische Zwecke als besonders geeignet: In erster Linie ist Twitter motivierend, da viele Prominente (oder deren KommunikationsberaterInnen) mit ihren Accounts präsent sind. Wenn es darum geht, den SchülerInnen Einblick in das politische Spektrum zu ermöglichen, sind PolitikerInnen auf keiner anderen SNS so gut vertreten wie auf Twitter. Zudem sind im Fall von Twitter die lexikalische, syntaktische und vor allem textuelle Komplexität durch die Prägnanz und Simplifizierung der Nachrichten (die Länge der Tweets ist auf 240 Zeichen begrenzt) sehr gering gehalten. Anders als ausführlichere Nachrichtenformate politischer Kommunikation (Zeitungen,

Kapelari, Suzanne (Hg.), Vierte „Tagung der Fachdidaktik“ 2019:

„Interdisziplinäre fachdidaktische Diskurse zur Bildung für nachhaltige Entwicklung“

(C) 2020 innsbruck university press, ISBN 978-3-99106-019-2, DOI 10.15203/99106-019-2 
Blogbeiträge, usw.) ist das Leseverständnis der meisten Tweets bereits ab dem Niveau „selbständige Sprachanwendung“ (B1-B2 des Gemeinsamen Europäischen Referenzrahmen für Sprachen (GER) (Trim et al., 2001) möglich. Allerdings sind sehr hohe kulturelle Kompetenzen erforderlich, da die Bedeutung von Tweets von medialen und politischen Kontexten, sowie von der kommunikativen Situation (Stalnaker, 2002) abhängig ist.

Wie in anderen Medien auch ist die politische Kommunikation über Twitter von impliziten Diskursstrategien geprägt (Garassino, Masia, \& Brocca, 2019; Lombardi-Vallauri \& Masia, 2014a). Implizite Kommunikation ist zwar aufgrund der Sprachökonomie für die tägliche Kommunikation notwendig, weil der Sender davon ausgehen kann, dass dem Empfänger gewisse Inhalte (wie die kommunikative Situation, die Beziehung zwischen Sender und Empfänger, die Informationen, die sie teilen und ihre kommunikative Intention) bereits bekannt oder zumindest nicht diskussionsbedürftig sind. Allerdings kann implizite Kommunikation auch angewendet werden, wenn ein Inhalt diskussionsbedürftig oder weniger bekannt ist und der Sender eine gewisse Botschaft vermitteln möchte, ohne der Kritik der Empfänger ausgesetzt zu sein (Lombardi-Vallauri \& Masia, 2014b). Wenn Informationen implizit übermittelt werden, minimiert der Sender zudem seine Verantwortung für den Wahrheitswert der Information, weil er für nichts zur Verantwortung gezogen werden kann, worüber er nichts explizit gesagt hat (Sbisà, 2007). Von diesen letzten Merkmalen der impliziten Kommunikation profitiert die Sprache der Politik, wenn implizite Kommunikationsstrategien angewendet werden, um den Empfänger zu manipulieren: Der Sender strebt nicht nach einem tieferen und genaueren Verständnis der Situation, sondern er möchte andere Menschen davon überzeugen, einen anderen Standpunkt einzunehmen, der für ihn selbst vorteilhaft ist, oder sie daran zu hindern, eine gewisse Meinung zu vertreten. Die wahren Absichten des Senders bleiben dem Empfänger verborgen (Buzărnescu, 2005: 154).

Das Risiko besteht nun darin, dass gewisse Inhalte vom Empfänger unbewusst angenommen werden und unterschwellig einen Weg in sein Hintergrundwissen

Kapelari, Suzanne (Hg.), Vierte „Tagung der Fachdidaktik“ 2019:

„Interdisziplinäre fachdidaktische Diskurse zur Bildung für nachhaltige Entwicklung“

(C) 2020 innsbruck university press, ISBN 978-3-99106-019-2, DOI 10.15203/99106-019-2 
finden. Studien zur impliziten Kommunikation (Drai \& de Saussure, 2016) haben gezeigt, dass implizit übermittelte Inhalte sich leicht ins Gedächtnis einprägen, weil sie weniger bewusst und eher unterschwellig verarbeitet werden (Lombardi Vallauri \& Masia, 2014a; Sbisà, 2007). Mehrere Autoren (de Saussure, 2013; Kerbrat-Orecchioni, 1986; Pinker, Nowak, \& Lee, 2008) haben hierbei argumentiert, dass Empfänger weniger dazu neigen, den Wahrheitsgehalt von impliziten Inhalten zu bewerten, als wenn diese explizit vermittelt werden.

Implizite Kommunikation kann mittels unterschiedlicher Formen und Strategien geschehen. In diesem Beitrag wird die Untersuchung impliziter Kommunikation auf zwei kommunikative Mittel beschränkt, die besonders relevant sind und bereits ausführlich erforscht wurden (de Saussure, 2013; Ducrot, 1972; KerbratOrecchioni, 1986; Lombardi Vallauri, 2002; Reboul, 2011; Sbisà, 2007; Stalnaker, 1973). Diese Mittel sind Präsuppositionen und Implikaturen.

Nach Stalnaker (1973) ist eine Präsupposition die Information, die als gemeinsame Grundlage für Sender und Empfänger vor einem kommunikativen Akt angenommen wird. Das passiert typischerweise, wenn Sender und Empfänger gemeinsames Vorwissen teilen. Vorausgesetzte Informationen werden durch Präsuppositionen übermittelt. Präsuppositionen bedienen sich typischer lexikalischer Ausdrücke oder syntaktischer Konstruktionen als Präsuppositionsauslöser. Typische Präsuppositionsauslöser sind z.B. definite Kennzeichnungen, iterative Verben, additive Adverbien, Zustandsveränderungsverben, faktive Verben und adverbiale Nebensätze. Ein Beispiel zu jedem dieser Präsuppositionsauslöser wird hier gegeben (Levinson, 1983, deutsche Fassung 2000,S. 197-201):

(1) Definite Kennzeichnungen: Die Schokoladenschachtel ist in der Küche.

(2) Iterative Adverbien: Dein Hund hat meine Vase wieder zerbrochen.

(3) Additive Adverbien: Jane trinkt auch Milch zum Frühstück.

(4) Zustandsveränderungsverben: Mark fängt wieder an, nachts Geräusche zu machen. 
(5) Faktive Verben: Mark erkannte, dass er hoch verschuldet war.

(6) Adverbiale Nebensätze: Als meine Tochter ihren Abschluss machte, kauften wir ihr ein neues Auto.

Ein weiteres Mittel, um implizite Informationen $\mathrm{zu}$ übermitteln, ist die Implikatur. Nach Grice (1975) werden Implikaturen als beabsichtigte Bedeutungen definiert, die nicht direkt aus der Oberflächenstruktur einer Äußerung ableitbar sind, sondern nur indirekt auf der Grundlage von Kontextinformationen und Annahmen des Weltwissens abgeleitet werden können, wie im folgenden Beispiel deutlich wird:

(7) Emmanuel Macron, 28.02. 2017 über Twitter: „Dans une économie de la transformation radicale que nous sommes en train de vivre, il faut prendre des risques."

[dt.: „In einer von radikalen Veränderungen geprägten Wirtschaft, die wir gerade erleben, müssen wir Risiken eingehen." ]

Die Bedeutung dieses Tweets (also, von welchen Risiken die Rede ist und wer sie eingehen sollte) ist den heutigen LeserInnen nicht so offensichtlich wie den Followers von Macron während des Wahlkampfs 2017, da sich in der Äußerung „il faut prendre des risques“ eine Implikatur befindet. Nur mit adäquaten Kontextinformationen kann der Empfänger verstehen, dass Macrons Botschaft impliziert, dass die damalige Regierung von François Hollande ${ }^{3}$ kein Risiko eingegangen ist, um von den wirtschaftlichen Veränderungen $\mathrm{zu}$ profitieren, bzw. dass François Hollande nicht mutig genug war.

Traditionell (Levinson, 1983: 183) werden zwei Arten von Implikaturen unterschieden: konversationelle und konventionelle Implikaturen. Konventionelle Implikaturen basieren auf der logisch-semantischen Bedeutung einer Äußerung: Ein typisches Beispiel für ihren Auslöser ist die Konjunktion , aber' wie in der folgenden Äußerung:

3 Staatspräsident der Französischen Republik vor Manuel Macron.

Kapelari, Suzanne (Hg.), Vierte „Tagung der Fachdidaktik“ 2019:

„Interdisziplinäre fachdidaktische Diskurse zur Bildung für nachhaltige Entwicklung“

(C) 2020 innsbruck university press, ISBN 978-3-99106-019-2, DOI 10.15203/99106-019-2 
(8) Johannes ist reich, aber sympathisch.

Durch die adversative Bedeutung der Konjunktion ,aber' wird unterstellt, dass alle reichen Menschen unsympathisch sind. Konventionelle Implikaturen sind Inferenzen aus der Bedeutung bestimmter lexikalischer Ausdrücke (Levinson, 1983). Im Gegensatz zu konversationellen Implikaturen erfordert ihr Verständnis lediglich die Sprachkompetenz des Empfängers, ein tieferes situatives Hintergrundwissen ist nicht nötig. Bei konversationellen Implikaturen hingegen kann die kommunikative Intention des Senders nur durch die richtige Einschätzung der kommunikativen Situation verstanden werden: Dafür müssen Sender und Empfänger Vorwissen und informative Ziele teilen. Konversationelle Implikaturen können z.B. aus indirekten Aussagen oder Antworten entstehen:

(9) A: Möchtest du einen Kaffee haben?

B: Ich wollte gleich ins Bett gehen.

In diesem Fall kann A verstehen, dass B keinen Kaffee möchte, selbst wenn seine Frage nicht direkt verneint wurde, weil die Gesprächspartner das Wissen teilen, dass Kaffee das Einschlafen erschwert. Das Beispiel zeigt, wie man in einer konversationellen Implikatur „mehr meinen kann, als tatsächlich [wörtlich] gesagt wurde“ (Levinson 1983, Dt. 2000: 107).

Wie auch in anderen Bereiche der Pragmatik (Brocca, Borowiec, \& Masia, 2020: S.84; Nuzzo, 2013) wird die implizite Kommunikation kaum in sprachdidaktischen Werken behandelt und es bleibt so an der Intuition der Lernenden, pragmatische Strategien richtig zu erschließen und zu verwenden. Dies kann jedoch sprachspezifisch sehr unterschiedlich zum Ausdruck kommen. Das Erkennen und Verstehen impliziter Informationen ist in österreichischen Lehrplänen ausdrücklich festgehalten, allerdings nur in Bezug auf die muttersprachliche Bildung: 
Textkompetenz: Texte reflektieren und bewerten: durch das Erkennen von Strategien der Beeinflussung politische Mündigkeit erlangen; die Fähigkeit der Perspektivenübernahme und der kritischen Betrachtung von Perspektiven fördern. (Lehrpläne für Deutsch) (Bundesministerium für Unterricht und Kunst, 1985)

Auch im GER (Gemeinsamem europäischen Referenzrahmen für Sprachen) wird implizite Kommunikation kaum behandelt (Trim et al., 2001). Erst ab dem Niveau C1 und bei „langen und komplexen“ Texten wird die Kompetenz, implizite Inhalte zu erkennen, berücksichtigt.

Argumentation Verstehen: C1: [der Lernende] kann ein weites Spektrum langer, komplexer Texte, denen man im gesellschaftlichen, beruflichen Leben oder in der Ausbildung begegnet, verstehen und dabei feinere Nuancen auch von explizit oder implizit angesprochenen Einstellungen und Meinungen erfassen. (Trim et al., 2001)

Da kaum Erfahrungswerte vorhanden sind, wie die Didaktisierung impliziter Kommunikationsstrategien umzusetzen ist, lohnt es sich, einen Blick auf die spontane Erkennung und Behandlung von Implizitem im Alltag zu werfen. Tatsächlich werden auf Implizitem aufgebaute Argumentationen von EmpfängerInnen oft abgewehrt, indem das Implizite offengelegt wird. Im folgenden Beispiel aus der Sprache der Politik attackierte der damaliger Innenminister Matteo Salvini einen Journalisten, der den sechzehnjährigen Sohn Salvinis im Urlaub auf einem Wasserscooter der Polizei entdeckte.

(10) Matteo Salvini 01.08. 2019, Interview in Milano Marittima: "Vada a riprendere i bambini, lei che è specializzato e le piace tanto.“ [dt.: „Gehen Sie Kinder filmen, da Sie darin spezialisiert sind und es Sie so sehr freut."]

In dieser Aussage wird durch eine konversationelle Implikatur (die auf einer Übergeneralisierung aufbaut) der Journalist diskreditiert: Der Journalist hat 
einen Minderjährigen gefilmt. Durch eine explizite Inferenz wird behauptet, dass er generell Minderjährige filmen würde, was unterschwellig den Vorwurf der Pädophilie vermittelt. Für diesen implizit ausgedrückten Inhalt - die ein unkritischer Empfänger leicht als wahr akzeptieren könnte - kann der Minister nicht verantwortlich gemacht werden. Würde der Minister diesen Inhalt hingegen durch eine Assertion ausdrücken, würde die Übertreibung direkt deutlich und die ZuhörerInnen würden den Inhalt der Aussage als solche ablehnen. Der Journalist offenbart die Implikatur:

(11) Journalist: "Mi sta dando del pedofilo?”
[dt.: „Unterstellen Sie mir, pädophil zu sein?“]

Der Innenminister muss nun die Assertion ,Der Journalist ist pädophil verneinen, da die Äußerung offenbar falsch ist und er für diese Äußerung verantwortlich (und ggf. strafbar) wäre.

(12) Matteo Salvini: "No, dico solo [...]" [dt.: "Nein, ich sage nur..."]

Der Journalist hat die Implikatur seines Angreifers paraphrasiert und dabei die implizit mitvermittelte Bedeutung explizit gemacht, was eine grundlegende Veränderung der Gesprächssituation provoziert.

Ein ähnlicher Prozess kann auch in didaktischen Kontexten angewendet werden. Die Fachdidaktik nutzt bereits Paraphrasierungsaufgaben, um Lernprozesse nachzuverfolgen und Diagnosen über das Textverständnis zu treffen. Sbisà (2007) berichtet von positiven Erfahrungen bei der Erkennung von impliziten Informationen durch das Paraphrasieren von Texten, vorausgesetzt, dass in der Paraphrase die impliziten Informationen explizit wiedergegeben werden. Die o.a. Beispiele (8) und (9) könnten folgendermaßen paraphrasiert werden:

(13) Meistens sind reiche Leute unsympathisch. Aber Johannes ist reich und sympathisch. 
(14) A: Möchtest du einen Kaffee haben?

B: Nein, ich sollte und werde keinen Kaffee trinken, weil ich gleich ins Bett gehen will, und wenn ich Kaffee trinke, kann ich schlecht einschlafen.

Natürlich sind die Paraphrasierungen (13) und (14) im Alltag zwecklos und verstoßen gegen pragmalinguistische Regeln; hier werden sie lediglich zwecks der Übersichtlichkeit angeführt. Hingegen ist die Paraphrasierung von Informationen mit manipulativer Intention, wie im Dialog (10-12), der bewährteste Weg, um eine kritische Position gegenüber Informationen aufzubauen. Nur wenn eine Information asseriert wird, ist es möglich die kommunikative Intention des Senders $\mathrm{zu}$ verstehen (Sbisà, 2007) und entsprechend zu reagieren.

\section{Darstellung didaktischer Materialien}

In diesem Abschnitt werden nun didaktische Materialien präsentiert, die als Beispiel für eine kritische Medienbildung im Rahmen des Fremdsprachunterrichts Französisch eingesetzt werden können. Die Materialien decken die Themenbereiche des aktuellen Zeitgeschehens ab und ermöglichen einen Blick auf die politische Landschaft Frankreichs. Sie dienen auch dem bewussten Umgang mit impliziten Kommunikationsstrategien in digitalen und analogen Medien und erhöhen damit die rezeptive und produktive pragmalinguistische Kompetenz. Am Ende der Einheit sollten die SchülerInnen in der Lage sein, implizite Informationen zu erkennen und umzudeuten, sowie eine entsprechende Reaktion zu formulieren. Mit dem Erkennen und Verstehen von impliziten Bedeutungen erlangen die Lernenden höhere textuelle Kompetenzen in der Fremdsprache. Eine Sensibilisierung für persuasive Kommunikation in anderen Sprachen und Textsorten ist ebenfalls zu erwarten.

Die rezeptiven Kompetenzen setzen sprachlich B2-Niveau nach GER voraus, während in der Produktionsphase sprachliche Kompetenzen des Niveaus B1 die

Kapelari, Suzanne (Hg.), Vierte ,Tagung der Fachdidaktik“ 2019:

„Interdisziplinäre fachdidaktische Diskurse zur Bildung für nachhaltige Entwicklung“

(C) 2020 innsbruck university press, ISBN 978-3-99106-019-2, DOI 10.15203/99106-019-2 
Mindestanforderung sind. Damit eignen sich die Materialien thematisch und sprachlich für die Sekundarstufe II (z.B. in der 12. Schulstufe) nach mindestens vier oder fünf Jahren Fremdsprachunterricht. Die Materialien können auch in einem fachübergreifenden Unterrichtssetting oder im bilingualen Sachfachunterricht im Kontext politischer Bildung, in Deutsch oder in einem Workshop im Bereich der digitalen Medien verwendet werden. Die angewendete Methode folgt dem CLIL Ansatz (Leisen, 2015), da das Material in einem Medien- und Politik-Workshop in der Fremdsprache eingesetzt wird. Sowohl die Bereitstellung authentischer Materialien als auch die Relevanz der behandelten Inhalte regen die Produktionsarbeit auf natürliche Weise an. Neben Authentizität und Aktualität besteht der hohe Wert von CLIL-Unterricht darin, dass durch ihn eine besondere Förderung von Textkompetenz ermöglicht wird (Mentz, 2004), da eine ständige Verknüpfung von Textrezeption und -produktion stattfindet.

Der vorgeschlagene Unterricht teilt sich in drei aufeinander aufbauende Phasen: Einstieg, Erarbeitung und Sicherung. In der Einstiegsphase wird den SchülerInnen ein Text präsentiert, in dem implizit übertragene Informationen enthalten sind. Durch ein induktives Verfahren werden die SchülerInnen angeleitet die impliziten Aussagen herauszuarbeiten. Als Textgrundlage wurde eine Rede von Emmanuel Macron gewählt (s. Materialien im Anhang 1a). Der französische Präsident wandte sich in einer Fernsehansprache am 18. Dezember 2018 an die französischen BürgerInnen, um die Maßnahmen nach den Demonstrationen der Gelbwestenbewegung (Fr. mouvement des Gilets jaunes) anzukündigen. ${ }^{4}$ Nach der Aktivierung von Vorwissen werden die SchülerInnen mit einer ausführlicheren Analyse konfrontiert. Es gilt herauszufinden, ob gewisse Informationen (s. Anhang 1b) im Text erhalten sind. Dafür können die SchülerInnen die schriftliche Version des Textes bearbeiten. Abgesehen von einem Distraktor (einer Aussage, die nicht im Text vorhanden ist), sind alle

4 Das gesamte Video kann unter folgendem Link angesehen werden: https:/www.elysee.fr/emmanuel-macron/2018/12/10/adresse-du-president-de-la-republiquedu-lundi-10-decembre-2018 (12.06.2019)

Kapelari, Suzanne (Hg.), Vierte „Tagung der Fachdidaktik“ 2019:

„Interdisziplinäre fachdidaktische Diskurse zur Bildung für nachhaltige Entwicklung“

(C) 2020 innsbruck university press, ISBN 978-3-99106-019-2, DOI 10.15203/99106-019-2 
erfragten Items durch Implikaturen oder Präsuppositionen übermittelt. Nach dem Lösen der Aufgabe werden die SchülerInnen gefragt, ob die gefundenen Informationen direkt vermittelt oder im Text, versteckt ${ }^{\star}$ sind. Das Beantworten dieser Fragen führt in eine kurze, induktive Informationsphase im Plenum, in der die SchülerInnen Metakompetenzen zu impliziten Kommunikationsstrategien anwenden und ein Bewusstsein für implizite Kommunikation entwickeln. Die SchülerInnen sollten danach in der Lage sein, zu erkennen, dass das Verständnis von impliziten Kommunikationsstrategien die Grundlage für gelungene Kommunikation ist (Sbisà, 2007, S. 6). In dieser Phase können z.B. typische Präsuppositionsauslöser erkannt und aufgelistet werden.

In der Erarbeitungsphase werden die SchülerInnen aufgefordert, selbst implizite Botschaften zu assertiven Äußerungen umzuwandeln und dazu eine kritische Stellungnahme zu formulieren. Der Bezug zur Lebenswelt kann hier durch Verweis auf die Interviewvorbereitung kritischer JournalistInnen oder politischer BeraterInnen oder der Einrichtung eines Fact-Checking Blogs (Brocca, Masia \& Will, in Vorbereitung) hergestellt werden. SchülerInnen werden ausgewählte Tweets vorgelegt, die Implikaturen oder Präsuppositionen beinhalten. Durch die Paraphrasierung der Tweets sollte die implizite Botschaft anfechtbar werden, wie in folgendem Beispiel verdeutlicht wird:
(15)
Jean-Luc Mèlenchon 03.05. 2017 über Twitter: „Il faut une éthique républicaine sans faille dans la police!“
[dt.: „Man braucht einwandfreie Verfassungsloyalität in der Polizei“]

Die Äußerung beinhaltet eine Implikatur, die durch ein verbe impersonnel ausgelöst wird. „Il faut“ (dt. es ist notwendig, man braucht) impliziert, dass das Objekt „une éthique républicaine sans faille“ nicht vorhanden ist. Eine mögliche Paraphrasierung durch eine Assertion könnte folgendermaßen lauten: 


\section{(16) Die Verfassungstreue der Polizei hat Lücken.}

Je nach Reife des didaktischen Kontexts sind mehrere kritische Auseinandersetzungen möglich wie z.B. ,Waren einige Polizisten nicht loyal oder sind sie generell nicht verfassungsloyal gewesen?' Weitere Beispiele werden tabellarisch in Anhang 2 dargestellt.

In der Sicherungsphase sollen die SchülerInnen das Erlernte in einem freieren Kontext anwenden können. Mithilfe von SNS mit pädagogischem Hintergrund und höheren Sicherheitsstandards, wie z.B. Fakebook oder Twiducate ${ }^{5}$ (Alvermann, 2017), könnten die SchülerInnen in einer geschlossenen Gruppe die Erfahrung von politischen Diskussionen in SNS erleben. Den SchülerInnen können hier auch durch Eckdaten von fiktiven BürgerInnen Rollen zugeteilt werden. Sie können dann ihr fiktives Profil in der Zielsprache ausführlicher bearbeiten und online mit PartnerInnen teilen. Anschließend könnten sie in einem Rollenspiel gewisse Positionen vertreten. Dadurch können die Lernenden auf die sprachliche Produktion fokussieren, ohne ihre eigene Idee vertreten zu müssen, und sie werden nicht gezwungen, ihre politische Orientierung zu offenbaren (bzw. eine eigene zu übernehmen). Die SchülerInnen werden in dieser Phase dazu angeregt, keine impliziten Äußerungen in ihre Argumente einzubauen bzw. die impliziten Botschaften anderer MitschülerInnen zu dementieren oder zu hinterfragen. Das lexikalische und textuelle Material der vorherigen Phasen kann und soll in der Textproduktion (Posts oder Tweets) wiederverwendet werden.

Der in dieser Phase mögliche thematische Anknüpfungspunkt an das kommunikative Verhalten in Debatten und Konfliktgesprächen on- und offline (Bundeszentrale für die politische Bildung, 2018; Fluter, 2017) bietet Raum für Aufklärung über die potentielle Aggressivität von Äußerungen mit impliziten Diskursmitteln (Janetzko, 2017; Piskorska, 2017). Es sollte dabei klar gestellt

5 Fakebook und Twiducate sind jeweils unter folgenden Adressen aufzurufen https://www.classtools.net/FB/home-page und https://www.livelingua.com/twiducate/.

Kapelari, Suzanne (Hg.), Vierte „Tagung der Fachdidaktik“ 2019:

„Interdisziplinäre fachdidaktische Diskurse zur Bildung für nachhaltige Entwicklung“

(C) 2020 innsbruck university press, ISBN 978-3-99106-019-2, DOI 10.15203/99106-019-2 
werden, dass, selbst wenn Meinungen mit Fakten belegt sind oder direkt asseriert werden, Meinungsdivergenzen unvermeidlich sind. Die Abschaffung impliziter Kommunikation soll nicht zum Ziel werden, weil (i) implizite Kommunikation unvermeidbar bzw. notwendig ist und (ii) explizite Kommunikation nicht immer das geeignete Instrument ist, konfliktfreie Kommunikation zu etablieren. Die Lernenden sollten ,lediglich“ befähigt werden, implizite Kommunikationsmittel zu erkennen, vor allem, wenn diese manipulatorische Zwecke verfolgen, und eine Abwehrstrategie (z.B. das Paraphrasieren und Hinterfragen von Aussagen) entwickeln, um andere Menschen und deren persönliche Meinung besser zu verstehen (Sbisà, 2007, S. 201). Somit soll es den SchülerInnen letztlich leichter fallen, ihre eigene Meinung zu bilden und diese respektvoll zu äußern.

\section{Fazit}

Die Rolle von SNS im fachdidaktischen Diskurs zur Bildung für nachhaltige Entwicklung ist erheblich. SNS stellen eine Chance für die heutigen Generationen dar, weil sie den Kommunikationsfluss beschleunigen und neue Formen der Kommunikation einführen. Sie finden auch in der politischen Kommunikation zunehmend Anwendung und haben einen starken Einfluss auf die junge Generation (Stodt, Wegmann, \& Brand, 2015). Trotzdem können die vom Medium bediente Geschwindigkeit und Knappheit die Komplexitätsreduzierung legitimieren; diese kann wiederum mit Manipulationsabsichten angewendet werden. Die Bewusstmachung von Manipulationstechniken sowie der Erwerb hinterfragender Lesestrategien verbessern die Rezeption von SNS und stärken gleichzeitig die demokratische Teilhabe im Sinne der SDGs. Die Frage, ob und wie ihre Rolle in der Unterrichtspraxis thematisiert werden soll, ist zugleich eine didaktische und eine gesellschaftliche Frage.

Ziel der allgemeinbildenden höheren Schulen Österreichs ist laut den Lehrplänen, junge Menschen zu befähigen, mündige Bürger eines demokratischen und von Digitalisierung beeinflussten Systems zu werden:

Kapelari, Suzanne (Hg.), Vierte „Tagung der Fachdidaktik“ 2019:

„Interdisziplinäre fachdidaktische Diskurse zur Bildung für nachhaltige Entwicklung“

(C) 2020 innsbruck university press, ISBN 978-3-99106-019-2, DOI 10.15203/99106-019-2 
Digitale Kompetenz, Medienkompetenz sowie politische Kompetenzen [...] haben das Ziel eines informierten, souveränen und verantwortlichen Umgangs mit Medien und Technik durch mündige Bürgerinnen und Bürger in der Demokratie und einer zunehmend von Digitalisierung beeinflussten Gesellschaft. Im Mittelpunkt steht dabei die reflektierte Verwendung von Medien [...]. (Bundesministerium für Unterricht und Kunst, 1985: Fassung vom 01.09.2018)

$\mathrm{Zu}$ den Kompetenzen, die Jugendliche auf eine digitale Gesellschaft vorbereiten, gehört die Fähigkeit, Nachrichten aus SNS kritisch zu lesen und vertrauenswürdige bzw. wahre Informationen aus dem Netz zu filtern. Der Fremdsprachenunterricht mit CLIL-Ansatz kann einen günstigen Bezugsrahmen für die Förderung der reflektierten Medienbildung darstellen, u.a. weil der Fremdsprachenunterricht eine emotionale Distanz $\mathrm{zu}$ politischen Themen ermöglicht, die im muttersprachlichen Unterricht ggf. schwieriger aufrecht zu erhalten wäre. In der kritischen Auseinandersetzung mit Medien soll die Analyse der impliziten Kommunikation eine wichtige Rolle übernehmen, weil diese es ermöglicht, die SchülerInnen zu befähigen, in SNS implizit vermittelte Informationen $\mathrm{zu}$ entschlüsseln und damit Inhalte tiefgreifender $\mathrm{zu}$ verstehen, logisch nachzuvollziehen oder kritisch zurückzuweisen. Die Analyse der impliziten Kommunikation ist wissenschaftlich auf einer soliden pragmalinguistischen Basis gegründet und gleichzeitig praxisorientiert und kann in unterschiedlichen Stufen und Fächern geübt und regelmäßig eingesetzt werden.

Die Beispiele in Abschnitt 4 haben im Französischunterricht für die Sekundarstufe II auf das Verstehen von politischen Botschaften fokussiert, indem ein altersangemessenes Thema (politische Kommunikation) mit den nötigen fremdsprachlichen Kompetenzen in Übereinstimmung gebracht ist. Neben sprachlichen und (inter-)kulturellen Zielen versucht dieser Beitrag auch ein fächerübergreifendes Ziel in Anlehnung an die Lehrpläne (s. Zitat oben) zu erreichen: Die aktive politische Teilhabe der SchülerInnen nachhaltig zu fördern und es ihnen zu ermöglichen, verantwortungsbewusste BürgerInnen in der 
digitalen Demokratie zu werden. Des Weiteren versucht dieser Aufsatz einen Beitrag dazu zu leisten, diese Generation zukunftsfähig zu machen und sie zu befähigen, Manipulation online zu erkennen und abzuwehren.

Die Kompetenzen im Umgang mit impliziten Kommunikationsstrategien können auch im früheren Alter Anwendung finden, wenn die fremdsprachlichen Herausforderungen reduziert werden und die Themen altersgerecht gewählt sind. Die Themen können weitere Bereiche über das Verständnis von politischer Kommunikation hinaus abdecken, von der impliziten Kommunikation in der Werbung bis hin zu Konflikten in privaten Gesprächen. Positive Wirkungen können so im Vermeiden von verbaler Aggression oder Cyber-Mobbing erwartet werden, da das Erkennen von impliziter Kommunikation helfen kann, andere $\mathrm{zu}$ verstehen und $\mathrm{zu}$ respektieren, sowie seine eigene Meinung zum Ausdruck zu bringen und diese selbstbewusst zu vertreten. Das ist nicht nur ein Ziel für eine nachhaltige Bildung, sondern auch für eine nachhaltige gesellschaftliche Entwicklung. ${ }^{6}$

\section{Anhang}

Anhang 1: Beispiel einer Didaktisierung einer politischen Rede. Mögliche Antworten der SchülerInnen sind kursiv geschrieben. Die Auslöser von impliziten Inhalten sind zwecks Übersichtlichkeit für Lehrkräfte fett markiert.

6 Das dargestellte Beispiel wird künftig an konkrete Klassensituationen adaptiert und getestet. Interessiertes Lehrpersonal wird herzlich eingeladen die Materialien mit zu entwickeln und zu erproben. Der Autor freut sich auf Kommunikation unter

Mail: nicola.brocca@uibk.ac.at

Twitter: @nicola_brocca

https://uibk.academia.edu/nicolabrocca

Kapelari, Suzanne (Hg.), Vierte „Tagung der Fachdidaktik“ 2019:

„Interdisziplinäre fachdidaktische Diskurse zur Bildung für nachhaltige Entwicklung“

(C) 2020 innsbruck university press, ISBN 978-3-99106-019-2, DOI 10.15203/99106-019-2 
a) Le discours d'Emmanuel Macron face aux gilets jaunes. 10 décembre 2018

"Françaises, Français,

[...] Nous avons tous vu le jeu des opportunistes (definite Kennzeichnung $\rightarrow>$ Präsupposition) qui ont essayé de profiter des colères sincères pour les dévoyer. Nous avons tous vu les irresponsables politiques (definite Kennzeichnung $\rightarrow>$ Präsupposition) dont le seul projet était de bousculer la République, cherchant le désordre et l'anarchie. Aucune colère ne justifie qu'on s'attaque à un policier (konversationelle Implikatur), à un gendarme, qu'on dégrade un commerce ou des bâtiments publics. Notre liberté n'existe que parce que chacun peut exprimer ses opinions, que d'autres peuvent ne pas les partager sans que personne n'ait à avoir peur de ces désaccords (konversationelle Implikatur). Quand la violence se déchaîne, la liberté cesse (Zustandsveränderungsverb $\rightarrow$ Präsupposition). C'est donc désormais le calme et l'ordre républicain qui doivent régner (Faktiver Verb und Zeitabverb -> Präsupposition) pourçarien ne se construira de durable tant qu'on aura des craintes pour la paix civile.

Ce sont quarante années de malaise qui ressurgissent: malaise des travailleurs qui ne s'y retrouvent plus (Zustandsveränderungsadverb à Präsupposition); malaise des territoires, villages comme quartiers où on voit les services publics se réduire (Zustandsveränderungsverb $\rightarrow$ Präsupposition) [...]. Nous voulons bâtir (Zustandsveränderungsverb $\rightarrow$ Präsupposition) une France du mérite, du travail, une France où nos enfants vivront mieux que nous. Cela ne peut se faire que par une meilleure école, des universités, de l'apprentissage et des formations qui apprennent aux plus jeunes et aux moins jeunes ce qu'il faut pour vivre libre et travailler. Nous voulons une France où l'on peut vivre dignement de son travail (konversationelle Implikatur).

Je reviendrai m'exprimer devant vous pour vous rendre compte. Mon seul souci, c'est vous; mon seul combat, c'est pour vous. Notre seule bataille, c'est pour la France.

Vive la République, vive la France!"

Textauszug aus:

https://www.elysee.fr/emmanuel-macron/2018/12/10/adresse-du-president-de-la-republique-du-lundi-10decembre-2018

Kapelari, Suzanne (Hg.), Vierte „Tagung der Fachdidaktik“ 2019:

„Interdisziplinäre fachdidaktische Diskurse zur Bildung für nachhaltige Entwicklung“

(C) 2020 innsbruck university press, ISBN 978-3-99106-019-2, DOI 10.15203/99106-019-2 
b) Après avoir lu le discours du President Macron, décide quell message n'est pas transmis par le texte:

1. Il y a des opportunistes.

....Nous avons tous vu le jeu des opportunistes .....

2. Des manifestants en colère ont attaqué un policier.

.... Aucune colère ne justifie qu'on s'attaque à un policier .....

3. Pour le moment, il n'y a pas de calme.

... C'est donc désormais le calme que doit régner ...

4. Pour le moment, il y a des craintes pour la paix civile.

...rien ne se construira de durable tant qu'on aura des craintes pour la paix civile...

5. Les milieux sociaux se sont unis.

Nicht vorhanden / pas trasmis

6. Actuellement, la France n'est pas un pays méritocratique.

...Nous voulons bâtir une France du mérite...

c) Comment ces informations sont-elles transmises? 
Anhang 2: Beispiele von Tweets, die implizite Informationen beinhalten, mögliche Paraphrasierung und mögliche kritische Nachfragen.

\begin{tabular}{|c|c|c|c|}
\hline Originaler Tweet & $\begin{array}{l}\text { Finden der } \\
\text { Implizite }\end{array}$ & Paraphrasierung & $\begin{array}{l}\text { Kritische } \\
\text { Nachfragen }\end{array}$ \\
\hline $\begin{array}{l}\text { “L'agriculture bio } \\
\text { est plus chère. Si } \\
\text { on augmente les } \\
\text { salaires, les gens } \\
\text { pourront acheter } \\
\text { de meilleurs } \\
\text { produits. “Jean- } \\
\text { Luc Mèlenchon } \\
03.15 .2017\end{array}$ & $\begin{array}{l}\text { „L'agriculture bio } \\
\text { est plus chère. Si } \\
\text { on augmente les } \\
\text { salaires, les gens } \\
\text { pourront acheter } \\
\text { de meilleurs } \\
\text { produits.“ } \\
\text { Durch eine } \\
\text { konversationelle } \\
\text { Implikatur wird } \\
\text { implizit die } \\
\text { Information } \\
\text { übertragen, dass } \\
\text { Bioprodukte für } \\
\text { die Konsumenten } \\
\text { besser sind als } \\
\text { konventionelle } \\
\text { Erzeugnisse. Die } \\
\text { bessere Qualität } \\
\text { der Bioprodukte } \\
\text { sowie die } \\
\text { Korrelation von } \\
\text { Einkommen und } \\
\text { Konsum von Bio- } \\
\text { Produkten wird als } \\
\text { selbstverständlich } \\
\text { unterstellt. }\end{array}$ & $\begin{array}{l}\text { „Wenn wir die } \\
\text { Löhne erhöhen, } \\
\text { können sich die } \\
\text { Menschen Bio- } \\
\text { Produkte leisten. } \\
\text { Bio-Produkte sind } \\
\text { besser. " }\end{array}$ & $\begin{array}{l}\text { Sind Bio-Produkte } \\
\text { wirklich besser? } \\
\text { Wie genau? Sind } \\
\text { sie nachhaltiger? } \\
\text { Gesünder? } \\
\text { Warum? } \\
\text { Konsumieren die } \\
\text { Menschen mehr } \\
\text { Bio-Produkte, } \\
\text { wenn sie mehr } \\
\text { verdienen? }\end{array}$ \\
\hline
\end{tabular}




\begin{tabular}{|c|c|c|c|}
\hline $\begin{array}{l}\text { "Le patriotisme } \\
\text { économique } \\
\text { protègera nos } \\
\text { entreprises } \\
\text { stratégiques, et } \\
\text { celles qui } \\
\text { subissent une } \\
\text { concurrence } \\
\text { déloyale!" Marin } \\
\text { e Le Pen } \\
03.13 .2017\end{array}$ & $\begin{array}{l}\text { „Le patriotisme } \\
\text { économique } \\
\text { protègera nos } \\
\text { entreprises } \\
\text { stratégiques, et } \\
\text { celles qui } \\
\text { subissent une } \\
\text { concurrence } \\
\text { déloyale! '.. } \\
\text { Durch einen } \\
\text { restriktiven } \\
\text { Relativsatzt wird } \\
\text { eine } \\
\text { Präsupposition } \\
\text { ausgelöst. Es wird } \\
\text { implizit die } \\
\text { Information } \\
\text { vermittelt, dass } \\
\text { ausländische } \\
\text { Unternehmen } \\
\text { unlauteren } \\
\text { Wettbewerb } \\
\text { betreiben. }\end{array}$ & $\begin{array}{l}\text { „Es gibt } \\
\text { ausländische } \\
\text { Unternehmen, die } \\
\text { unlauteren } \\
\text { Wettbewerb } \\
\text { betreiben.“ }\end{array}$ & $\begin{array}{l}\text { Was ist } \\
\text { unlauterer } \\
\text { Wettbewerb? } \\
\text { Wer macht es? } \\
\text { Betreibt } \\
\text { Frankreich auch } \\
\text { unlauteren } \\
\text { Wettbewerb im } \\
\text { Ausland? Würde } \\
\text { ein europaweiter } \\
\text { Mindestlohn den } \\
\text { Wettbewerb } \\
\text { reduzieren? }\end{array}$ \\
\hline $\begin{array}{l}\text { „Le vrai } \\
\text { patriotisme } \\
\text { économique, c'est } \\
\text { d'abord d'être } \\
\text { compétitif“' } \\
\text { Macron } \\
03.13 .2017 \\
\text { Als Antwort auf } \\
\text { den vorherigen } \\
\text { Le Pens Tweet. }\end{array}$ & $\begin{array}{l}\text { „Le vrai } \\
\text { patriotisme } \\
\text { économique, c'est } \\
\text { d'abord d'être } \\
\text { compétitif. “ } \\
\text { Der Modifikator } \\
\text { „le vrai“ impliziert } \\
\text { (konventionelle } \\
\text { Implikatur), dass } \\
\text { es auch falschen } \\
\text { Patriotismus gibt. }\end{array}$ & $\begin{array}{l}\text { „Es gibt falschen } \\
\text { ökonomischen } \\
\text { Patriotismus (den } \\
\text { von Le Pen). “ }\end{array}$ & $\begin{array}{l}\text { Warum ist } \\
\text { Kompetitiv-sein } \\
\text { patriotisch? } \\
\text { Warum ist der } \\
\text { andere } \\
\text { ökonomische } \\
\text { Patriotismus } \\
\text { falsch? Was } \\
\text { bedeutet } \\
\text { ökonomischer } \\
\text { Patriotismus? }\end{array}$ \\
\hline
\end{tabular}

Kapelari, Suzanne (Hg.), Vierte „Tagung der Fachdidaktik“ 2019:

„Interdisziplinäre fachdidaktische Diskurse zur Bildung für nachhaltige Entwicklung“

(C) 2020 innsbruck university press, ISBN 978-3-99106-019-2, DOI 10.15203/99106-019-2 


\section{Literatur}

BBWF, Bundesministerium für Bildung, Wissenschaft und Forschung (2019). Masterplan Digitalisierung. Abgerufen am 12.12.2019

https://bildung.bmbwf.gv.at/schulen/schule40/masterplan_digitalisierung_pi.pd f? $6 \mathrm{ma} 2 \mathrm{fg}$

Bundesministerium für Unterricht und Kunst, 1985. Gesamte Rechtsvorschrift für Lehrpläne - allgemeinbildende höhere Schulen, Fassung vom 01.09.2018. Verordnung des Bundesministers für Unterricht und Kunst vom 14. November 1984 über die Lehrpläne der allgemeinbildenden höheren Schulen;

https://www.ris.bka.gv.at/GeltendeFassung.wxe?Abfrage=Bundesnormen\&Ge setzesnummer $=10008568 \&$ FassungVom $=2018-09-01$

Alvermann, D. E. (2017). Social Media Texts and Critical Inquiry in a Post Factual Era. Journal of Adolescent \& Adult Literacy, 61, 338. doi:10.1002/jaal.694

Barberá, P., Jost, J. T., Nagler, J., Tucker, J. A., \& Bonneau, R. (2015). Tweeting From Left to Right: Is Online Political Communication More Than an Echo Chamber? Spychological Science, 26(10), 1531-1542. doi: https://doi.org/10.1177/0956797615594620

Baumgartner, P., Brandhofer, G., Ebner, M., Gradinger, P., \& Korte, M. (2016). Medienkompetenz fördern - Lehren und Lernen im digitalen Zeitalter. In M. Bruneforth, F. Eder, K. Krainer, C. Schreiner, A. Seel, \& C. Spiel (Eds.), Nationaler Bildungsbericht Österreich 2015 (Vol. Band 2 - Alle Analysen im Detail pp. 95-132). Graz: Leykam.

Bundesministerium für Bildung, Wissenschaft und Forschung (2019). Masterplan Digitalisierung. Abgerufen am 12.12.2019

https://bildung.bmbwf.gv.at/schulen/schule40/masterplan digitalisierung pi.pd f? $6 \mathrm{ma} 2 \mathrm{fg}$

Bundeszentrale für die politische Bildung (2018). Hate Speech. Abgerufen am 12.12.2019

http://www.bpb.de/lernen/digitale-bildung/medienpaedagogik/231236/hatespeech

Bitkom (2019). Mit 10 Jahren haben die meisten Kinder ein eigenes Smartphone. Abgerufen am 12.12.2019 von

https://www.bitkom.org/Presse/Presseinformation/Mit-10-Jahren-haben-diemeisten-Kinder-ein-eigenes-Smartphone

Brocca, N., \& Masia, V. (in Vorbereitung). Supporting reading-understanding of implicit communication. Experimental Evidences

Kapelari, Suzanne (Hg.), Vierte „Tagung der Fachdidaktik“ 2019:

„Interdisziplinäre fachdidaktische Diskurse zur Bildung für nachhaltige Entwicklung“

(C) 2020 innsbruck university press, ISBN 978-3-99106-019-2, DOI 10.15203/99106-019-2 
Brocca, Nicola; Borowiec, Ewa und Masia, Viviana (2020). Didactics of pragmatics as a way to improve social media liter-acy. An experiment proposal with Polish and Italian students in L2. In: heiEDUCATION Journal 5 |2010, S. 81-107

Brocca, N., Masia, V., \& Will, L. (in Vorbereitung). Make pragmatics great again! Teaching implicatures and presuppositions for democracy education in the age of Twitter and Facebook. Educating the Global Citizen: International Perspectives on Foreign Language Teaching in the Digital Age 28. März 2019, Ludwig-Maximilians-Universität München.

Buzărnescu, Ş. (2005). Public sociology - conceptual system and research methodology.

DAK-Gesundheit, \& UKE, Deutsches Zentrum für Suchtfragen am Universitätsklinikum Hamburg-Eppendorf (2018). WhatsApp, Instagram und Co. - so süchtig macht Social Media. DAK-Studie: Befragung von Kindern und Jugendlichen zwischen 12 und 17 Jahren. Befragung der forsa Gesellschaft für Sozialforschung und statistische Analysen GmbH, https://www.saferinternet.at/fileadmin/redakteure/Footer/Studien/dak-studiesocial-media-nutzung-1968596.pdf.

de Saussure, L. (2013). Background relevance. Journal of Pragmatics, 59, 189. doi:10.1016/j.pragma.2013.08.009

DiFonzo, N. (2011). The Echo-Chamber Effect. The New York Times. Abgerufen am 12.12.2019 von https://www.nytimes.com/roomfordebate/2011/04/21/barack-obama-and-thepsychology-of-the-birther-myth/the-echo-chamber-effect

Drai, N., \& de Saussure, L. (2016). Quand l'implicite devient explicite: d'un accident expérimental à une étude pilote. Syntaxe \& Sémantique, 17, 115-133.

Dubois, E., \& Blank, G. (2018). The echo chamber is overstated: the moderating effect of political interest and diverse media. Information, Communication \& Society, 1-17. doi:10.1080/1369118X.2018.1428656

Ducrot, O. (1972). Dire et ne pas dire : principes de sémantique linguistique. Paris: Hermann.

Fluter-Nr.63. (2017). Propaganda. Bonn: Juni 2017.

Garassino, D., Masia, V., \& Brocca, N. (2019). Tweet as you speech. The role of implicit strategies and pragmatic functions in political communication: Data from a diamesic comparison. In: RILA, Rassegna Italiana di Linguistica Applicata, Nr. 2-3-2019 - anno LI.

Grice, P. H. (1975). Logic and conversation. In P. Cole \& J. Morgan (Eds.), Syntax and Semantics 3: Speech Acts (pp. 41-58). New York: Academic Press.

Janetzko, D. (2017). Manipulation in sozialen Netzwerken. Deutschland und Europa, Zeitschrift für Gemeinschaftskunde, Geschichte und Wirtschaft 74-2017. Landeszentrale für Politische Bildung. Baden-Württemberg. (Die neuen

Kapelari, Suzanne (Hg.), Vierte „Tagung der Fachdidaktik“ 2019:

„Interdisziplinäre fachdidaktische Diskurse zur Bildung für nachhaltige Entwicklung“

(C) 2020 innsbruck university press, ISBN 978-3-99106-019-2, DOI 10.15203/99106-019-2 
Medien und die politische Meinungsbildung. »Fake News« - ein Produkt der neuen Medien?), 26-36. Abgerufen am 12.12.2019 von www.deutschlandundeuropa.de

JIM-Studie (2015). Jugend-Information-(Multi-)Media. Basisstudie zum Medienumgang 12- bis 19-Jähriger in Deutschland. Abgerufen am 12.12.2019 https://www.mpfs.de/studien/jim-studie/2015/

JIM-Studie (2016). Jugend-Information-(Multi-)Media. Basisstudie zum Medienumgang 12- bis 19-Jähriger in Deutschland. Abgerufen am 12.12.2019 https://www.mpfs.de/studien/jim-studie/2016/

JIM-Studie (2017). Jugend, Information, Medien. Basisuntersuchung zum Medienumgang 12- bis 19-Jährigen. Abgerufen am 12.12.2019 https://www.mpfs.de/studien/jim-studie/2017/

Jugend-Internet-Monitor (2019). Welche Sozialen Netzwerke nutzen Österreichs Jugendliche? . Saferinternet.at. Abgerufen am 12.12.2019 https://www.saferinternet.at/services/jugend-internet-monitor/

Kerbrat-Orecchioni, C. (1986). L' implicite: Paris : Colin.

KMK (2017). Bildung in der digitalen Welt. Strategie der Kultusministerkonferenz.

Leisen, J. (2015). Lehrmaterialien im CLIL-Unterricht. Zeitschrift für Interkulturellen Fremdsprachenunterricht, $20(<\mathrm{b}>\mathrm{E}-\mathrm{ISSN}:</ \mathrm{b}>1205-6545)$.

Levinson, S. C. (1983). Pragmatics (1. publ.. ed.): Cambridge [u.a.] : Cambridge Univ. Press.

Lombardi Vallauri, E. (2002). La struttura informativa dell'enunciato (1. ed.. ed.): [Firenze] : La Nuova Italia Ed.

Lombardi Vallauri, E., \& Masia, V. (2014a). Implicitness impact: measuring texts. Journal of Pragmatics, 61, 161-184.

Lombardi Vallauri, E., \& Masia, V. (2014b). Misurare l'informazione implicita nella propaganda politica italiana. In R. Librandi \& R. Piro (Eds.), L'italiano della politica e la politica per l'italiano. Atti del XI Convegno ASLI Associazione per la Storia della Lingua Italiana (Napoli, 20-22 novembre 2014). Firenze: Franco Cesati Editore.

Mentz, O. (2004). Bilingualer Unterricht mit der Zielsprache Französisch. Eine Untersuchung über die aktuelle Situation in Deutschland. In F.-J. Meißner, J. Mertens, \& M. Reinfried (Eds.), Französisch heute 35/2 (pp. 122-133).

Mills, K. L. (2016). Possible Effects of Internet Use on Cognitive Development in Adolescence. Media and Communication, 4(3), 4-12.

Mishra, P., \& Koehler, M. J. (2006). Technological Pedagogical Content Knowledge: A new framework for teacher knowledge. Teachers College Record, 108 (6), 1017-1054. 
Nuzzo, E. (2013). La pragmatica nei manuali d'italiano L2: una prima indagine sull'atto linguistico del ringraziare. Revista De Italianística, 2 (26) 5-29. Abgerufen am 12.12.2019 von https://doi.org/10.11606/issn.2238-8281.v2i26p5-29

Pariser, E. (2011). The filter bubble: what the Internet is hiding from you (1. publ.. ed.): London : Viking.

Pinker, S., Nowak, M. A., \& Lee, J. J. (2008). The logic of indirect speech. Proceedings of the National Academy of Sciences of the United States of America, 105(<b $>$ E-ISSN $:</ \mathrm{b}>1091-6490)$. doi:10.1073/pnas.0707192105

Piskorska, A. (2017). On the strength of explicit and implicit verbal offences: A relevance-theoretic view. In S. Bonacchi (Ed.), Verbale Aggression: Multidisziplinäre Zugänge zur verletzenden Macht der Sprache (pp. 51-72). Berlin, Boston: De Gruyter.

Reboul, A. (2011). A relevance-theoretic account of the evolution of implicit communication. Studies in Pragmatics, 13 1-19.

Sbisà, M. (2007). Detto non detto: le forme della comunicazione implicita (1. ed. ed.). Roma [etc.]: GLF editori Laterza.

Stalnaker, R. (1973). Presuppositions. Published under the Auspices of the Association for Symbolic Logic, $2(<\mathrm{b}>$ ISSN: $\quad</ \mathrm{b}>0022-3611), \quad 457$. doi:10.1007/BF00262951

Stalnaker, R. (2002). Common ground. Linguistics and Philosophy, 25, 701-721

Stodt, B., Wegmann, E., \& Brand, M. (2015). Geschickt geklickt?! Zum Zusammenhang von Internetnutzungskompetenzen, Internetsucht und Cybermobbing bei Jugendlichen und jungen Erwachsenen. Leipzig: VISTAS Verlag.

Sunstein, C. R. (2017). \#Republic: divided democracy in the age of social media. Princeton, Oxford: Princeton University Press.

Trim, J., North, B., \& Coste D. (2001). Gemeinsamer europäischer Referenzrahmen für Sprachen: lernen, lehren, beurteilen. Langenscheidt, 2001, ISBN 978-3-468-49469-7, goethe.de [abgerufen am 20. Dezember 2019]

Vereinigte Nationen (2015) Transformation unserer Welt: die Agenda 2030 für nachhaltige Entwicklung. Resolution 69/315, UN-Generalversammlung, 10. September 2015

Vergeer, M. (2013). From echo chamber to persuasive device? Rethinking the role of the Internet in campaigns. New media \& society., 15 (, 1461-7315), 127.

Kapelari, Suzanne (Hg.), Vierte „Tagung der Fachdidaktik“ 2019:

„Interdisziplinäre fachdidaktische Diskurse zur Bildung für nachhaltige Entwicklung“

(C) 2020 innsbruck university press, ISBN 978-3-99106-019-2, DOI 10.15203/99106-019-2 